\title{
PARALLEL MODEL BASED FAULT DETECTION ALGORITHM FOR ELECTRONIC PARKING BRAKE SYSTEM
}

\author{
B. J. MOON ${ }^{1)}$, H. G. JUNG ${ }^{2)}$, S. G. $\mathrm{LEE}^{3)}$ and D. H. $\mathrm{KIM}^{4)^{*}}$ \\ ${ }^{1)}$ Department of Automotive Engineering, Dae Duk College, Daejeon 305-715, Korea \\ ${ }^{2)}$ Department of Automotive Engineering, Han Yang University, Seoul 133-791, Korea \\ ${ }^{3)}$ Department of Mechanical Engineering, Kyung Hee University, Gyeonggi 446-701, Korea \\ ${ }^{4)}$ Department of Electrical Engineering, Kyung Hee University, Gyeonggi 446-701, Korea
}

(Received 5 November 2012; Revised 13 March 2013; Accepted 24 June 2013)

\begin{abstract}
This paper describes a parallel model-based fault detection algorithm for an electronic parking brake (EPB) system, which consists of an electronic control unit with built-in current sensor and braking force sensor. For the EPB system to supply sufficient parking force to a vehicle, the parking force sensor is of utmost importance. If a fault occurs in this sensor, sufficient parking force may not be supplied, thereby seriously threatening the safety of the vehicle. Thus, a fault detection method is required for the parking force sensor of the EPB system to improve the safety of vehicles. For this purpose, a highly reliable fault detection method is needed to detect abnormal fault signals, which cannot be detected by the existing on-line sensor monitoring fault detection methods. This paper proposes a novel parallel model-based fault detection algorithm for the EPB system, which compares the physical sensor data with the mathematical model, the fuzzy model, and the neural network model at the same time. In order to reduce false alarms, the magnitude of thresholds and the operation counts are changed adaptively. When the proposed parallel model-based fault detection algorithm detects severe failures of the force sensor, it warns the driver in advance to prevent accidents due to the failures. The proposed algorithm is verified by hardware-in-theloop simulations in various situations.
\end{abstract}

KEY WORDS : Electronic parking brake, Model-based fault detection, Mathematical model, Fuzzy model and neural network model

\section{INTRODUCTION}

The most severe test condition for the parking brake is that it must keep the vehicle stationary on the 30 percent road slope in gross vehicle weight states. Around $40 \mathrm{kgf}$ of human power is required for the hand-operated lever type brake, and $50 \mathrm{kgf}$ for the foot-operated pedal type brake (Moon et al., 2002). However, sometimes drivers, such as females or seniors, cannot supply sufficient operational power. Thus, cases have been reported in which accidents occurred after parking. To prevent these, the electronic parking brake (EPB) system, which generates parking force through a simple switch operation by the driver, is required.

Currently produced EPB can be largely classified into two types. The first is a cable puller type that uses motor to pull parking cable as shown in Figure 1, where the latter is a motor on caliper type that generates braking force by delivering the power directly from motor to caliper.

The cable puller type is divided once again into either a single puller type or a dual puller type depending on the structure of system. In the structure of single puller type,

*Corresponding author. e-mail: donghani@khu.ac.kr each cable is connected to the actuator and then two cables are connected through the equalizer. In contrast, two cables are directly connected to the actuator in the structure of dual puller type. Note that the single puller type is easier to install on a vehicle compared to the dual puller type. The single puller type is used in the EPB system of this paper, where its structure is shown in Figure 2 (Chung et al., 2008).

For an EPB system to supply sufficient parking force, a parking force sensor should measure the force of the EPB

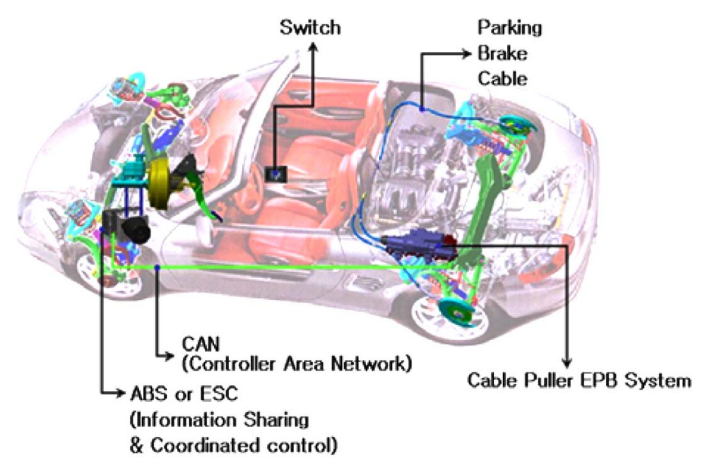

Figure 1. Configuration of the cable puller EPB system. 


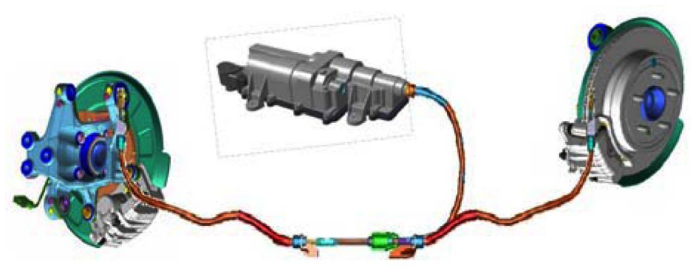

Figure 2. Single puller type EPB system.

system. If a fault occurs in this sensor, sufficient parking force may not be supplied, thereby seriously threatening the safety of the vehicle. Thus, a fault detection method is required for the parking force sensors of EPB systems to improve the safety of vehicles. For this purpose, a highly reliable model based fault detection method is needed to detect abnormal fault signals, which cannot be detected by the existing on-line sensor monitoring fault detection methods (Han et al., 2008). In the last decade of the 20th century, the field of fault detection has shown rapid progress due to safety demands in automotive industry. Besides, fault detection has become a must for many other industries due to productivity and quality considerations ('zero defects' manufacturing) (Albas et al., 2001).

The existing online sensor monitoring fault detection methods are limited because they only check some measurable output variables. Since the conventional approaches do not provide deeper insight and usually do not allow fault detection, several model-based fault detections are developed by using input/output signals and applying dynamic process models. The model-based fault detection algorithm predicts the future values of the states, outputs, and comparing them with the measured values (Sreedhar et al., 1995).

The model-based fault detection method can easily determine the defection in the system as shown in Figure 3. Residual is the difference between the actual system and the designed model. If there is no fault in the system and the model is precisely designed as the real system, the error value would be zero. However, the difference is not be zero in practical applications because the model is not based on the complete physical data (Isermann and Balle, 1997;

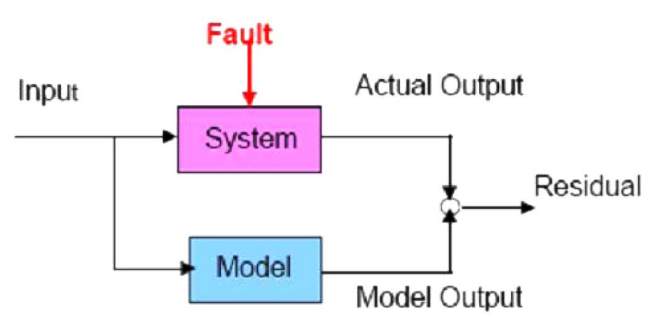

Figure 3. Block diagram of the model-based fault detection algorithm.

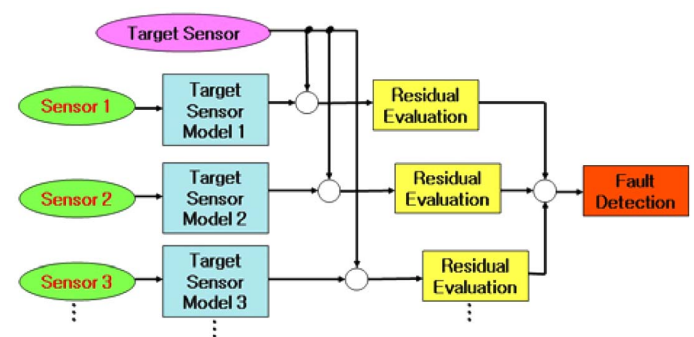

Figure 4. Structure of the multi model-based fault detection algorithm.

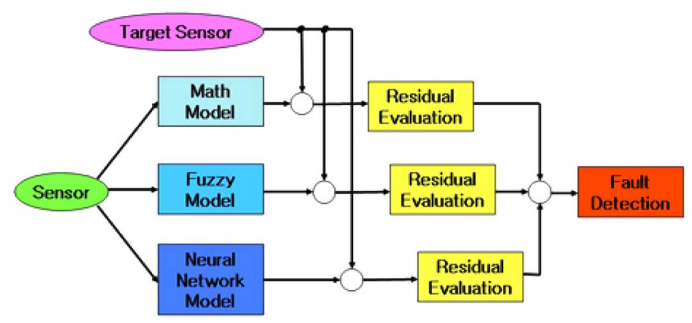

Figure 5. Structure of the parallel model-based fault detection algorithm.

Pfeufer, 1997). In the previous studies (Boskovic and Mehra, 1998; Maybeck and Stenvens, 1991; Gopinathan et al., 1998), the multi model-based fault detection algorithm has been widely used as shown in Figure 4. In order to detect faults existing in one sensor among a quantity of $n$ sensors, the proposed algorithm uses the rest of $n-1$ number of sensors to generate n-1 number of fault detection models. Note that the minimum number of sensors to apply this algorithm is three in this case. The reason for implementing many models for one sensor is that the possibility of a false alarm is high and it needs more time to determine faults if fault detection is conducted by using one model.

In this paper, the parallel model-based fault detection algorithm consisting of the mathematical model, the fuzzy model, and the neural network model is proposed to improve the reliability of fault detection as shown in Figure 5. The proposed algorithm can be applied to a system, where only one sensor could be implemented into the model. In addition, the adaptive thresholds and the operation counting method are applied to enhance the robustness against the system modeling errors, disturbances, and noises.

This paper is organized as follows. In Section II, modeling methods of three independent models are presented and the parallel model-based fault detection algorithm for EPB system is proposed. The proposed algorithm is verified by hardware-in-the-loop (HIL) simulation in Section III and concluding remarks follow in Section IV. 


\section{PARALLEL MODEL-BASED FAULT DETECTION ALGORITHM FOR ELECTRONIC PARKING BRAKE}

\subsection{Basic Structure}

To apply the parallel model-based fault detection algorithm for EPB system, three independent models - a mathematical model, a fuzzy model, and a neural network model - should be developed to estimate parking force using four input variables - an operating time, a voltage, a current, and a temperature. In the mathematical model, a parity space method (Patton and Willcox, 1987; Beard, 1971) is used, in which the input/output information is used to identify the consistency of system. However, it is necessary to have a higher order equation model to represent the parking force of the EPB, which is a nonlinear dynamic system. Due to the calculation time, the higher order equation model is difficult to be used in real applications. In case of the low order equation models, the result is not accurate enough.

The fuzzy model is determined through the following four stages: fuzzification, rule-based stage, inference, and defuzzification. For fuzzy inference, the maximumminimum method is used because it is assessed to be highly reliable and has been applied in many systems (Choi et al., 2005). For the defuzzifier, the center of gravity method is used (Moon et al., 2009). The fuzzy control algorithms and fuzzy inference systems present many advantages such as simplicity, robustness, no need to find transfer functions, nonlinear behavior, and adaptability (Altinten et al., 2003). Therefore, a fuzzy logic control system is installed to help operators make decisions regarding the control of a blast furnace. Researchers summarized, "There is a slight difference in knowledge between a multiple number of experts... In the case of expert systems which process ill-structured problems, an $100 \%$ success could not be possible (Iida et al., 1998)."

The neural network model is made by using a twolayered feed-forward network and learned by the LevenbergMarquardt (LM) back-propagation algorithm (Demuth et al., 2009). Artificial neural networks can usefully be applied in cases like this, where traditional mathematical modeling becomes complex due to nonlinear characteristics of the system (Bose and Raghavan, 2005). When the failure data with strong nonlinearity are given to the neural network model-based fault detection method, accurate diagnosis can be performed based on the trained patterns. In addition, reliable results can be obtained for the patterns, which have some sort of error. Thus, the neural network model has been actively used in the field of fault diagnosis (Sorsa and Koivo, 1993; Patton and Chen, 1994; Fussel et al., 1997; Sorsa, 1991). Like the above mentioned, each method has its own advantages over others, so they can be complementary to each other.

Figure 6 shows the process of the proposed parallel model-based fault detection algorithm for EPB system,

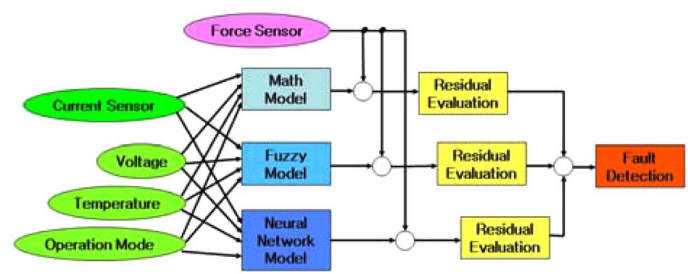

Figure 6. Structure of the parallel model-based fault detection algorithm for EPB system.

which can be summarized into four steps as follows:

(1) Obtain data for fault detection.

a) Measurement through force sensor.

b) Estimation of parking force using three models (the mathematical model, the fuzzy model, and the neural network model) with motor current, voltage, temperature, and operation mode of EPB system.

(2) Calculate residual by comparing the value of measured force and estimated force of each model.

(3) Collect fault information by using the output of evaluated residual through the magnitude of thresholds and the number of operations.

(4) Detect fault by using the fault information in Step 3.

If the EPB system is normal, the value of estimated parking force and the measured value will be identical. However, if the EPB system has some faults, the value of estimated parking force and the measured value will be different and thus, faults of the EPB system can be detected. A detailed description of the three models is described in the following sections.

\subsection{Mathematical Model}

The actuator in EPB system is composed of a DC motor, a gear box, a spindle, a force sensor module, and cables as shown in Figure 7. The motor generates torque and this torque is amplified through the deceleration box. Once the gear box receives the torque, it rotates and moves the spindle. The force generated at this time is divided through the main cable and is applied to the cables on both sides, as the same force. The force working on the brake pad then moves the parking force sensing spring in the parking force sensing module and, thereby, the force is transferred as voltage through the hall sensor at the force sensor module.

The mathematical model is derived from the elastic energy formula and the electric energy formula. Electric

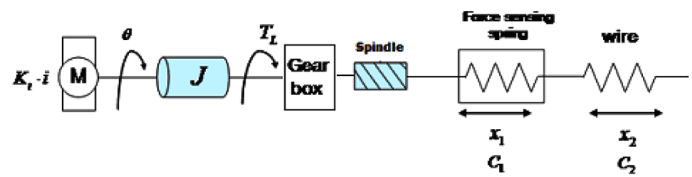

Figure 7. Actuator configuration of the cable puller EPB system. 
energy applied to the EPB system generates tension on the cables through the DC motor, gear box and spindle. This tension makes the spring to move at the force sensor module and is expressed as voltage through the hall sensor at the force sensor module. This system is most accurately represented by a nonlinear model. Since it is difficult to derive high order nonlinear model for the actual system, nonlinear systems are approximated as simple models.

Assuming that the applied electric energy is identical to the parking force energy output by changes in the spring, the elastic energy, E, and electric energy, $W_{S}$, are defined as follows:

$$
\begin{aligned}
& E=\frac{1}{2} k x^{2} \\
& W_{s}=V \cdot I \cdot T
\end{aligned}
$$

where $\mathrm{k}$ is the stiffness, $\mathrm{x}$ is the displacement, $\mathrm{V}, \mathrm{I}$, and $\mathrm{T}$ correspond to voltage, current, and time, respectively. When Hooke's law $(\mathrm{F}=\mathrm{k} \bullet \mathrm{x})$ is substituted into (1), the elastic energy is rewritten as follows:

$E=\frac{1}{2} k x^{2}=\frac{F^{2}}{2 k}=\beta \cdot W_{s}$

where $\mathrm{F}$ is the measured force and $\beta$ is the elastic energy constant. Based on (3), the estimated force, $F_{E}$, is expressed as follows:

$$
F_{E}=\alpha \cdot \bar{W}_{s}, \alpha=\sqrt{2 k \cdot \beta}
$$

where $\alpha$ is the model constant.

The precision of parking force sensor is decreased as the temperature changes. In other words, temperature change affects not only the characteristics of the sensor itself, but also the instrument area, where the sensor is installed. In addition, the changes in outputs with respect to temperature, which is actually occurring in the EPB system, are larger than those specified in the sensor specification (Mihov et al., 2004). In (4), estimated temperature is not considered in the parking force estimation models. Since residuals related to temperature change are large, large thresholds have to be set.

The fault detection model that does not consider temperature is insufficient. In this respect, the temperature constant, $\gamma$, is added in (4) to enhance the temperature effect and the reliability of model-based fault detection algorithm as follows:

$F_{r}=\alpha \cdot \gamma \cdot \sqrt{W_{S}}$

where $F_{T}$ is the estimated force, which reflects temperature changes. Note that $\gamma$ applied to the mathematical model, the fuzzy model, and the neural network model is based on Ohm's law (V=IR). Table 1 summarizes the sum of motor driving currents due to various temperatures as a lookup
Table 1. Motor current classified by temperature.

\begin{tabular}{cc}
\hline Temperature & Sum of motor currents \\
\hline$-40^{\circ} \mathrm{C}$ & $1351.2 \mathrm{~A}$ \\
$-20^{\circ} \mathrm{C}$ & $824.3 \mathrm{~A}$ \\
$0^{\circ} \mathrm{C}$ & $671.8 \mathrm{~A}$ \\
$25^{\circ} \mathrm{C}$ & $571.5 \mathrm{~A}$ \\
$45^{\circ} \mathrm{C}$ & $516.0 \mathrm{~A}$ \\
$65^{\circ} \mathrm{C}$ & $487.2 \mathrm{~A}$ \\
$85^{\circ} \mathrm{C}$ & $483.5 \mathrm{~A}$ \\
\hline
\end{tabular}

table. Note that the sum of motor driving currents is the accumulated value of currents measured in every $10 \mathrm{msec}$ during operation time, which is the MCU cycle time of EPB system. When temperature decreases, motor resistance and the resistance of individual parts decrease as well. Thus, more current flows at lower temperature while less current flows at higher temperature.

\subsection{Fuzzy Model}

The actuator For fuzzy model to perceive input values, input values must be normalized before being used. The fuzzification process becomes necessary in order to show these normalized input values as linguistic variables.

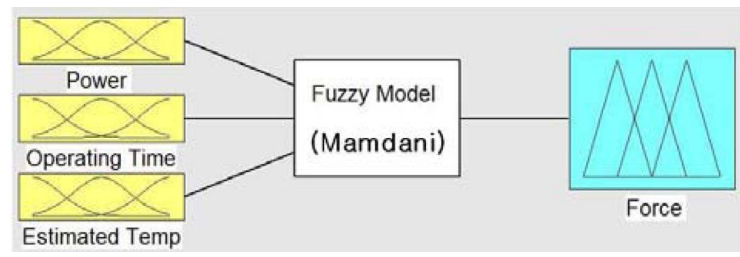

Figure 8 . Block diagram of the fuzzy model.

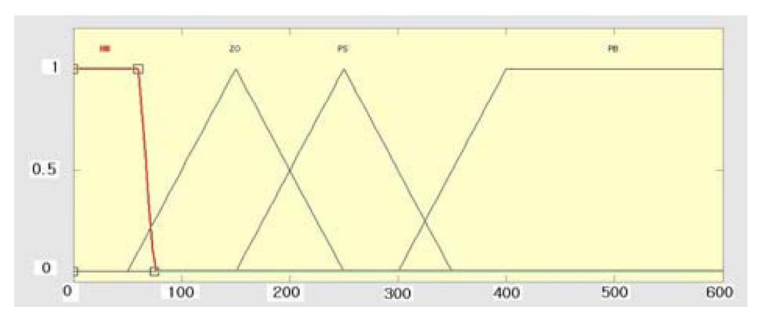

Figure 9. Membership functions of power.

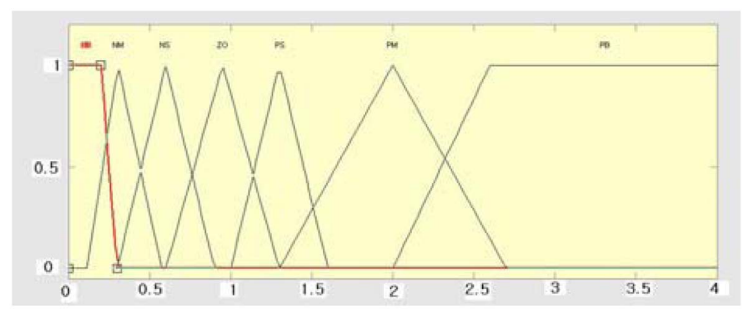

Figure 10. Membership functions of time. 


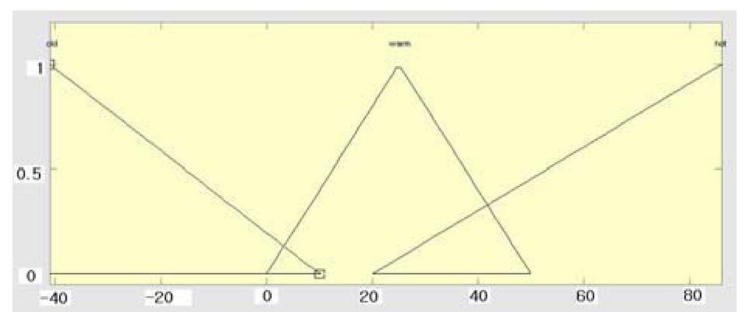

Figure 11. Membership functions of temperature.

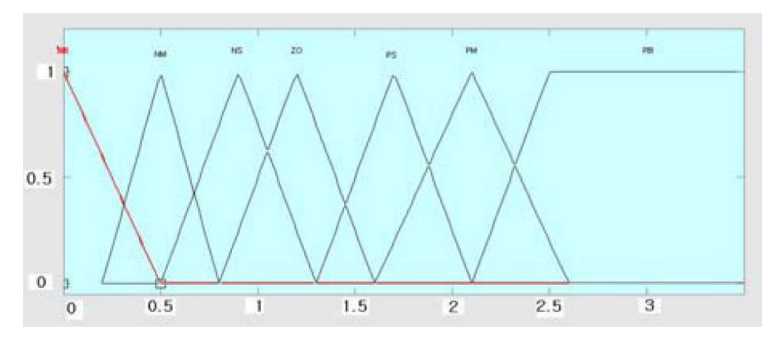

Figure 12. Membership functions of force.

Considering the advantages in calculation, triangular membership functions are used to implement fuzzification in this paper. The range of normalization for individual input and output variables is determined by using the experimental results from existing systems. The linguistic variables are used for both inputs and outputs to express them within the normalization range.

\subsubsection{Input values}

(a) Power

The linguistic variable of electricity is defined as a 'Power' and its value is normalized into the range of 0 to 600 as shown in Figure 9.

(b) Operating Time

The linguistic variable of run-time is defined as an 'Operating Time' and its value is normalized into the range of 0 to 4 as shown in Figure 10.

(c) Estimated Temperature

The linguistic variable of estimated temperature is defined as an 'Estimated Temp' and its value is normalized into the range of $-40^{\circ} \mathrm{C}$ to $85^{\circ} \mathrm{C}$, which is the operating temperature of EPB system as shown in Figure 11.

\subsubsection{Output values}

The linguistic variable of an estimated parking force of EPB system is defined as a 'Force' and its value is normalized into the range of 0 to 3.5 as shown in Figure 12.

The fuzzy rules are defined as follows:

i) If (Power is not PB) and (Operating Time is NM) and (Estimated Temp is warm), then (Force is $\mathrm{PB}$ )

ii) If (Power is not PB) and (Operating Time is NM) and (Estimated Temp is hot), then (Force is PB)

iii) If (Power is not PB) and (Operating Time is NM) and
(Estimated Temp is cold), then (Force is PM)

where PB, PM, PS, ZO, and NB, NM, NS correspond to positive big, positive medium, positive small, zero, and negative big, negative medium, negative small respectively.

The total number of fuzzy rules is 84 because the number of sub-fuzzy sets for input variables is divided into four for power, seven for operating time, and three for temperature. The maximum-minimum method is used as the fuzzy inference method because it is known to be highly reliable and has been applied in many different systems. Note that the center of gravity method is used for defuzzification.

\subsection{Neural Network Model}

The neural network used in this paper is a two-layer, feedforward network developed by using the MathWorks' neural network fitting tool (Demuth et al., 2009). The twolayer feed-forward network, which uses sigmoid transfer function in the hidden layer and linear transfer functions in the output layer, can appropriately adjust the problem of multi-dimensional mapping and can provide consistent data and sufficient neurons to the hidden layer. The network is learned by the LM back-propagation algorithm.

\subsubsection{Structure of neural network}

The structure of the neural network used in fault detection is shown in Figure 13, which consists of a hidden layer and an output layer in a $[4 \times 20 \times 1 \times 1]$ structure. Four input variables - operating time, voltage, currents, and temperature - are selected in a $4 \times \mathrm{N}$ (number of data) matrix, where output is the estimated value of parking force.

\subsubsection{Training of neural network}

The adjustment of connection strength is determined by the LM back-propagation learning algorithm and connection strength. Bias is adjusted so that the differences between actual outputs and targeted values are minimized. Neural network has been learned successfully when the value of mean squared error (MSE) is small.

Figure 14 shows the snapshot of neural network training, where 19 iterations of training are repeated until the validation error occurs 6 times. Figure 15 shows the training errors, validation errors, and test errors, which represent the performance of trained neural network. Note that the best validation performance of learning outcomes has appeared after 13 iterations. The experimental results

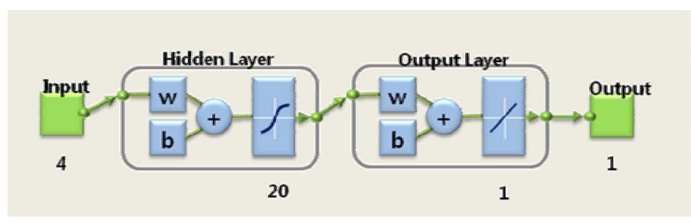

Figure 13. Two-layer feed-forward network. 


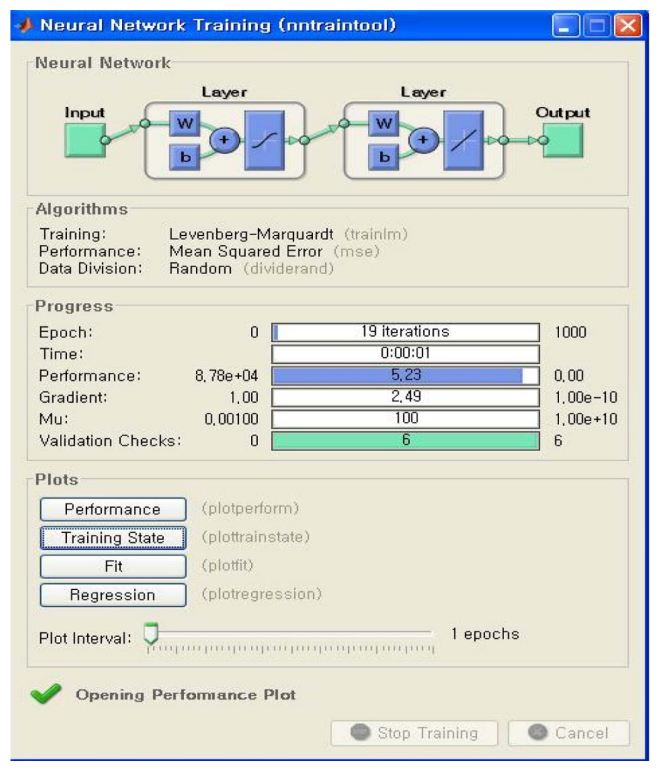

Figure 14. Snapshot of neural network training.

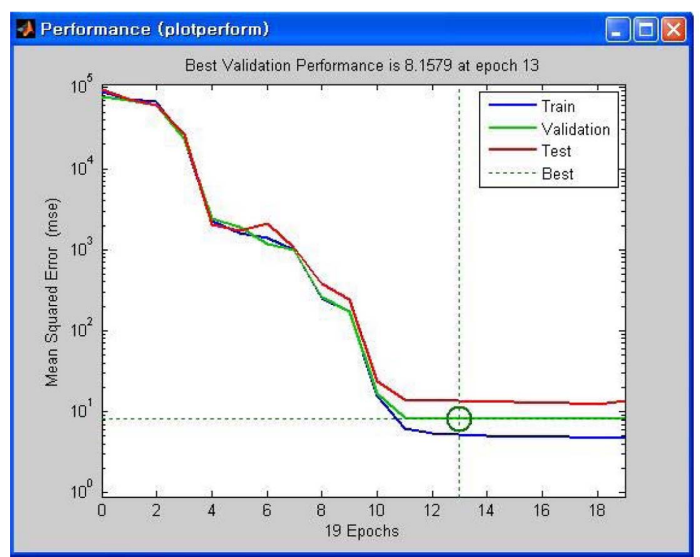

Figure 15. Results of training performance.

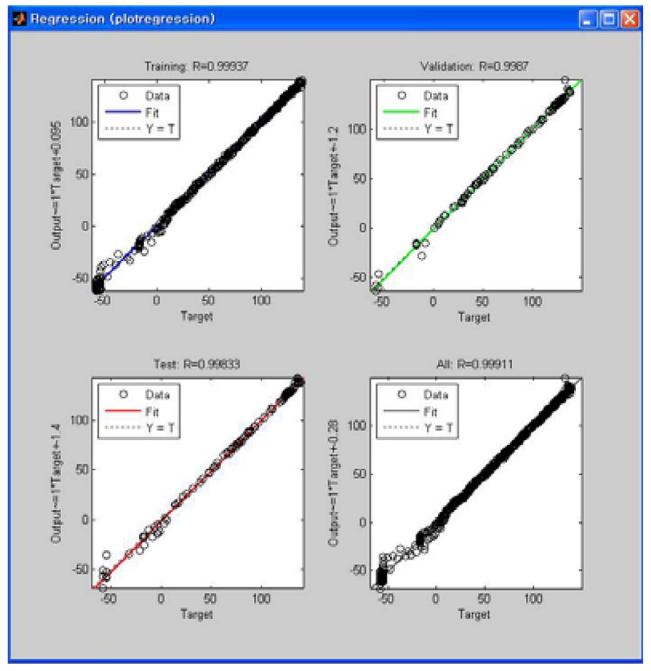

Figure 16. Results of training state. shown in Figure 15 are reliable because the final value of MSE is small, the validation errors and test errors have similar characteristics, and the overfitting has occurred in the 13th iteration, where the best learning results have validated.

The analysis of network response can be seen through a regression. Figure 16 shows the training states, which are the results of linear regression between the neural network outputs and the corresponding targets. As shown in Figure 16 , the training output, the testing output, and the validation output follow the target, where the overall response of regression value is higher than 0.999 . In this condition, we can conclude that the responsiveness of network is acceptable.

The trained neural network can be tested in MATLAB by using SIM functions as follows:

$$
\text { SIM_OUTPUT }=\operatorname{sim}(\text { net,NEW_Input); }
$$

\subsection{Adaptive Threshold}

An adaptive threshold method is used to implement a parallel model-based fault detection algorithm of the EPB system, which is strong against temperature changes. Fault detection using a constant threshold cannot reflect the relationship between temperature and the error of model. If the constant threshold is too low, the false alarm rate at normal states increases. In contrast, the actual fault might be missed if the threshold is set too high to avoid a false alarm. Thus, the adaptive threshold method with respect to temperature is expected to overcome the drawback of the constant threshold (Jung et al., 2007).

The standard deviation of consumed electric energy increases as the temperature decreases as shown in Table 1. Thus, the precision of model parameters should be higher in high temperature areas, whereas they should be lower in low temperature areas. In order to implement a fault detection algorithm that is strong against temperature changes, low thresholds are used in high temperature areas, whereas high thresholds are used in low temperature areas.

Table 2 shows the adaptive thresholds of the mathematical model for three different temperatures. The magnitude of the residual is $37 \%$ in the low temperature area, but the magnitude of adaptive threshold for fault detection is set to $43 \%$ and faults are not detected.

Table 2. Motor current classified by temperature.

\begin{tabular}{cccc}
\hline Temperature & Residual & $\begin{array}{c}\text { Adaptive } \\
\text { threshold }\end{array}$ & $\begin{array}{c}\text { Fault } \\
\text { detection }\end{array}$ \\
\hline Low & $37 \%$ & $43 \%$ & $\mathrm{~N}$ \\
Room & $37 \%$ & $35 \%$ & $\mathrm{Y}$ \\
High & $37 \%$ & $30 \%$ & $\mathrm{Y}$ \\
\hline
\end{tabular}

- Low temperature : $-20^{\circ} \mathrm{C} \sim-40^{\circ} \mathrm{C}$

Room temperature : $-20^{\circ} \mathrm{C} \sim 45^{\circ} \mathrm{C}$

High temperature : $45^{\circ} \mathrm{C} \sim 85^{\circ} \mathrm{C}$ 


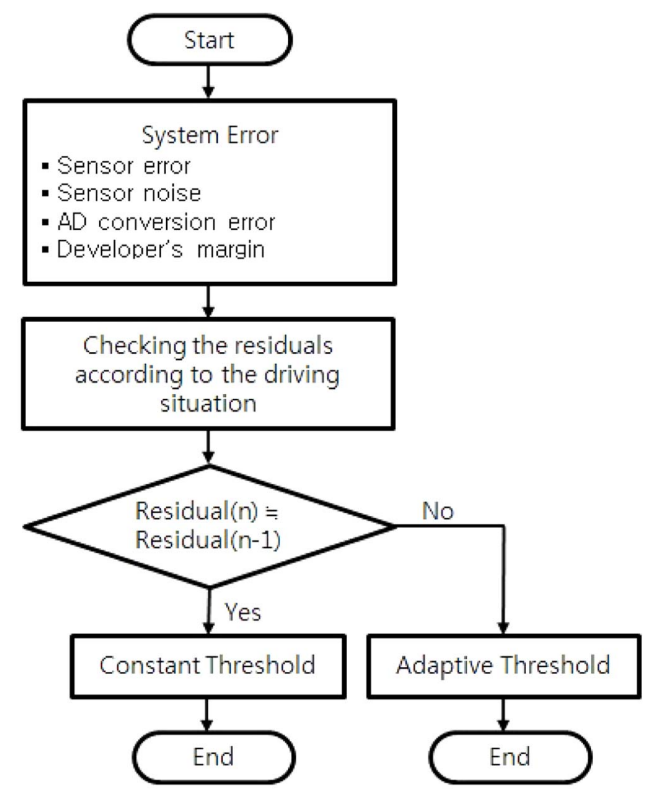

Figure 17. Flowchart of constant threshold and adaptive threshold.

However, in the high temperature area, although the magnitude of residual is $37 \%$, the magnitude of adaptive threshold for fault detection is set to $35 \%$ and faults are detected. In this way, the adaptive threshold method is applied to the parallel model-based fault detection algorithm of the EPB system by using low threshold values in high temperature areas with high accuracy of a model and high threshold values in low temperature areas with low accuracy of a model.

The error of braking force at the EPB system is $15 \%$, which is the sum of parking force sensor error $(10 \%)$, the sensor noise $(1 \%)$, the micro-computer's AD conversion error $(1 \%)$, and the developer's margin $(3 \%)$. The error values of individual models are measured based on the load cells when the absolute values of parking force are measured. The error of mathematical model is $20 \%$, the error of the fuzzy model is $10 \%$, and the error of the neural network model is $5 \%$.

Figure 17 shows the flowchart of determining either constant threshold or adaptive threshold. In order to determine the adaptive threshold, the basis system errors such as sensor error, AD conversion error, the developer's margin, etc. have to be obtained. The residual between the actual system and the proposed model is then computed according to the driving situation (temperature, driving method, etc.). The constant threshold is applied when the computed value of residual is small. However, the possibility of false alarm and non-detection increases as the computed value of residual increases. Thus, the deviation of residual must be taken into account according to the driving situation in order to determine the adaptive threshold. In this way, the proposed algorithm is able to
Table 3. Model errors and the threshold of fault detection at room temperature.

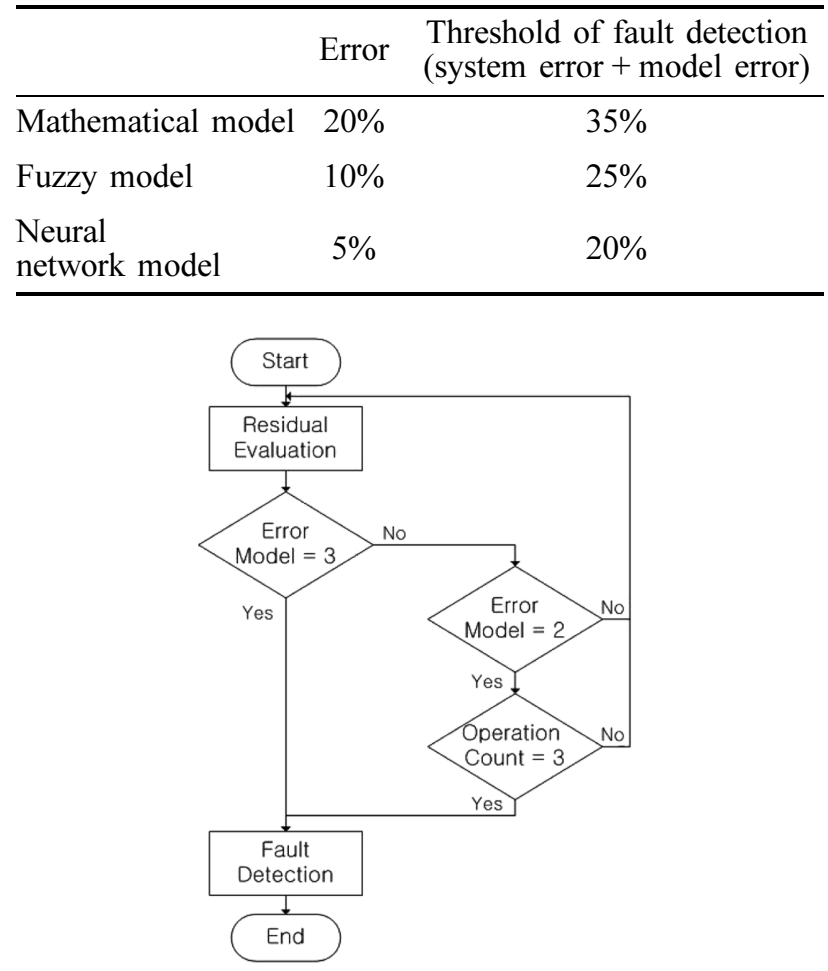

Figure 18. Flowchart of fault detection method to guarantee robustness.

Table 4. Model errors and the threshold of fault detection at room temperature.

\begin{tabular}{lcc}
\hline & Temperature & Adaptive threshold \\
\hline \multirow{2}{*}{ Mathematical } & Low & $43 \%$ \\
model & Room & $35 \%$ \\
& High & $30 \%$ \\
Fuzzy model & Low & $30 \%$ \\
& Room & $25 \%$ \\
Neural network & High & $22 \%$ \\
model & Low & $25 \%$ \\
& Room & $20 \%$ \\
\hline
\end{tabular}

detect the exact failure according to the driving situation.

Since the specified error values are the company's confidential information, random error values are used in the proposed algorithm.

2.6. Method to Secure the Robustness of Fault DetectionAdaptive Threshold

Figure 18 shows the flowchart of proposed fault detection algorithm to guarantee robustness of structure. As shown in 
Figure 18, residual evaluation step compares the three models (mathematical model, fuzzy model, and neural network model) and the outputs of parking force sensor. At this point, faults are detected when the residual exceeds the predefined threshold values shown in Table 4 and the corresponding number of models becomes more than three. When the number of models that exceeds the threshold is two, fault is detected after repeating the same procedure three times. Note that fault is not detected if there is only one model that exceeds the threshold.

\section{EXPERIMENTAL RESULTS}

Hardware-in-the-loop (HIL) simulation is used as a test method to verify the proposed parallel model-based fault detection algorithm. As shown in Figure 19, the HIL simulation uses parts of interest, or parts that cannot be mathematically analyzed in vehicles as hardware such as EPB, brake cable, and caliper. It also configures the remaining parts as vehicle models, thereby configuring software and hardware into a system to conduct simulations. The characteristics of HIL simulation include the ability to conduct the experiments that are identical to actual car environment at a reduced cost in comparison to vehicle tests, with shortened development time, and without adding the risk of actual drivers. In addition, HIL simulation offers the ability to repeat the conduct tests.

\subsection{Results of Mathematical Model Considering} Temperature

Figure 20 to Figure 22 show the experimental results for

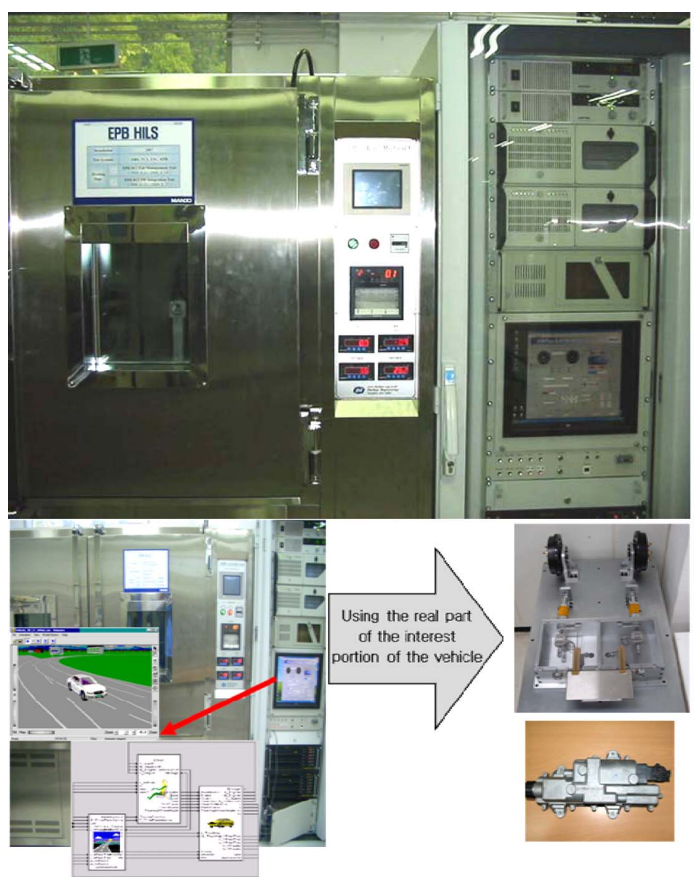

Figure 19. HIL simulation of the EPB system.

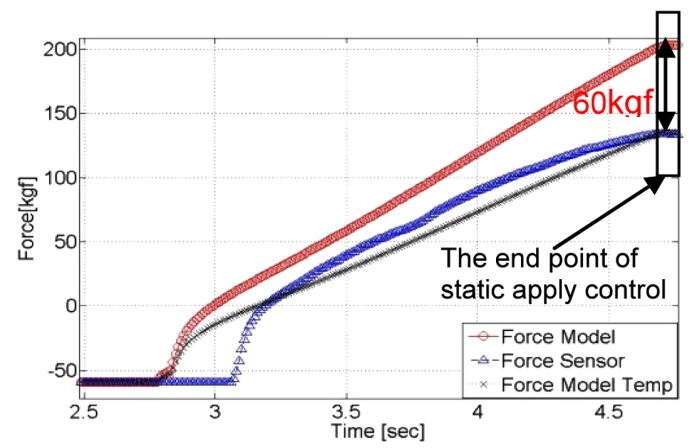

Figure 20. Results of mathematical model when temperature is and is not considered $\left(-40^{\circ} \mathrm{C}\right)$.

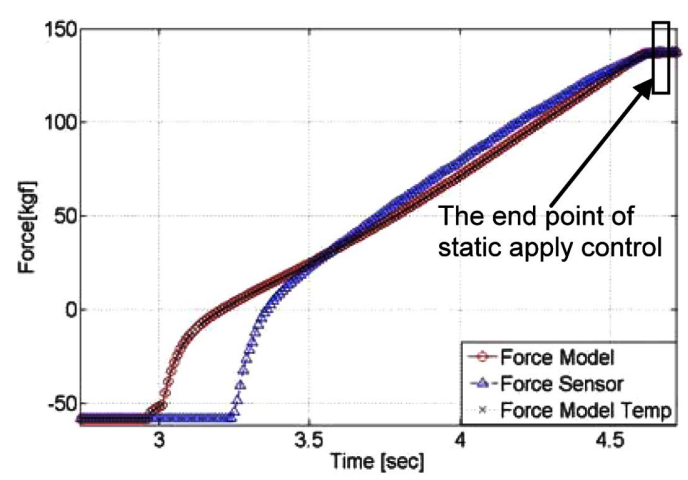

Figure 21. Results of mathematical model when temperature is and is not considered $\left(25^{\circ} \mathrm{C}\right)$.

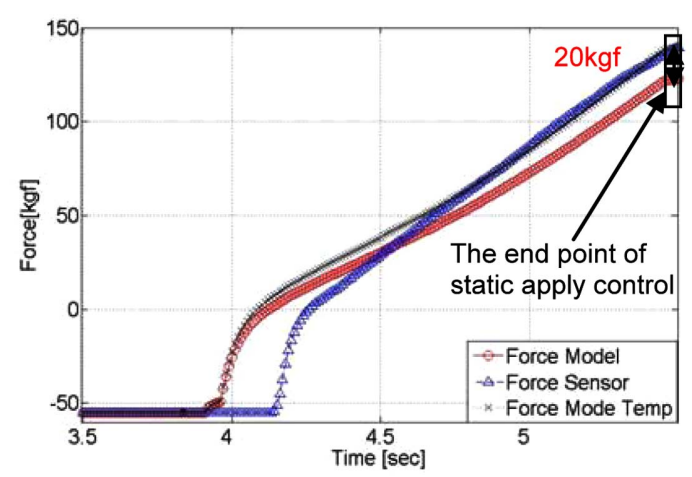

Figure 22. Results of mathematical model when temperature is and is not considered $\left(85^{\circ} \mathrm{C}\right)$.

three different temperatures when temperature was and was not considered in mathematical model. In these figures, 'Force Model' represents the results of mathematical model without adding temperature consideration, where the estimated temperature was considered in 'Force Model Temp'. In addition, the output of 'Force Sensor' was added by using HIL simulation.

As shown in Figure 20, the difference between 'Force 
Model' and the output of force sensor was approximately $60 \mathrm{kgf}$ when the low temperature $\left(-40^{\circ} \mathrm{C}\right)$ was not taken into account. However, the value of 'Force Model Temp' followed the output of force sensor because the estimated temperature was considered into the mathematical model. At room temperature, no particular differences were shown between 'Force Model', 'Force Model Temp', and 'Force Sensor' as shown in Figure 21.

When the high temperature $\left(85^{\circ} \mathrm{C}\right)$ was considered in the model, the difference between 'Force Model' and 'Force Sensor' was approximately $20 \mathrm{kgf}$ as shown in Figure 22. Similar to Figure 20, the value of 'Force Model Temp' that reflected estimated temperature properly followed the output of force sensor. Since the parking force models of the EPB system showed large output variations in accordance with temperature changes, temperature effects were considered in all three models (mathematical model,

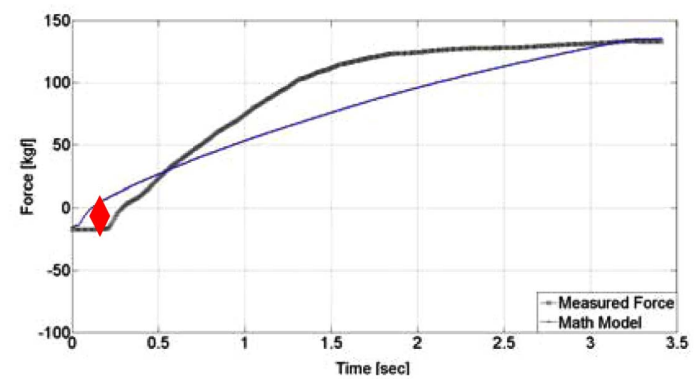

Figure 23. Results of measured force and mathematical model $\left(-40^{\circ} \mathrm{C}\right)$.

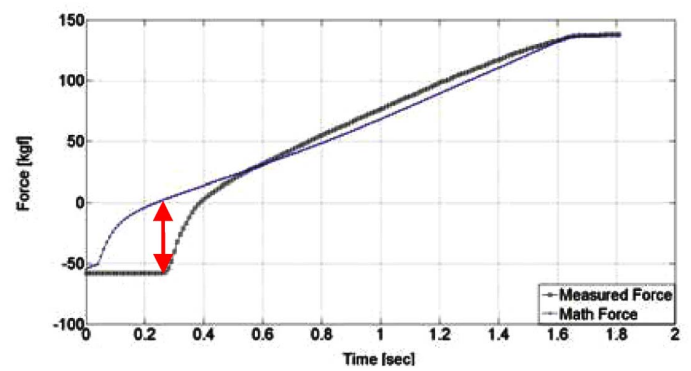

Figure 24. Results of measured force and mathematical model $\left(25^{\circ} \mathrm{C}\right)$.

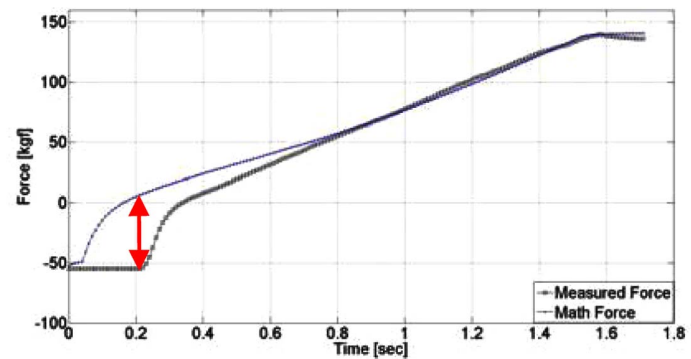

Figure 25. Results of measured force and mathematical model $\left(85^{\circ} \mathrm{C}\right)$. fuzzy model, and neural network model) of the proposed parallel model-based fault detection algorithm.

Figure 23 to Figure 31 show the HIL simulation results of the output values and the estimated values of the parking forces for mathematical model ('Math Force'), fuzzy model ('Fuzzy Model'), and neural network model ('Neural Network Force'). Based on the experimental results, the proposed model was able to follow the output values of actual parking force sensor, even at very low temperature $\left(-40^{\circ} \mathrm{C}\right)$ and at very high temperature $\left(85^{\circ} \mathrm{C}\right)$.

\subsection{Results of Mathematical Model}

The mathematical model of EPB system used in this paper was an approximated low order model. Thus, the mathematical model could not sufficiently reflect the nonlinearity of the EPB system in the initial operation time between $0 \mathrm{~ms}$ and $300 \mathrm{~ms}$. As shown in Figure 23 to Figure 25 , the actual output value of parking force sensor did not

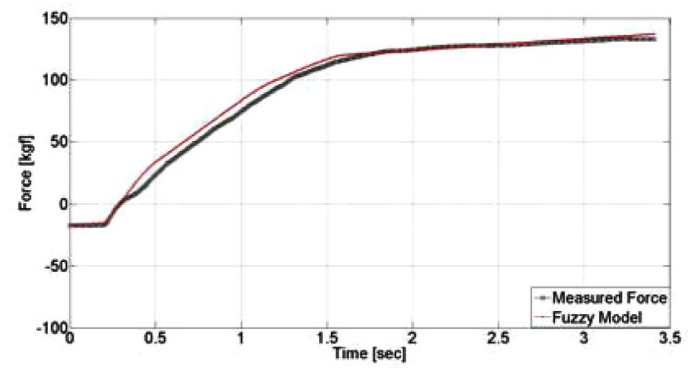

Figure 26. Results of measured force and fuzzy model $\left(-40^{\circ} \mathrm{C}\right)$

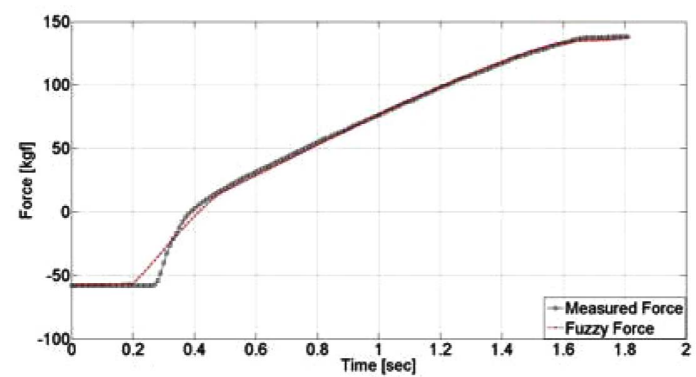

Figure 27. Results of measured force and fuzzy model $\left(25^{\circ} \mathrm{C}\right)$.

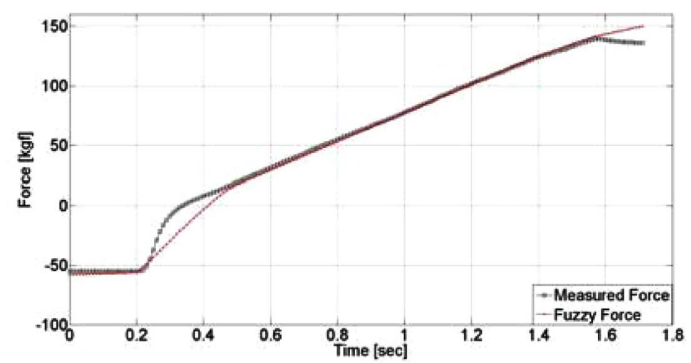

Figure 28. Results of measured force and fuzzy model $\left(85^{\circ} \mathrm{C}\right)$. 
change, whereas the mathematical model was changed, as indicated with red arrows.

\subsection{Results of Fuzzy Model}

The experimental results shown in Figure 26 to Figure 28 indicated that the fuzzy model was able to follow the output value of actual parking force sensor better than the mathematical model in the actual operation section of the EPB system $(-60 \mathrm{kgf} \sim 150 \mathrm{kgf})$ and various temperatures $\left(-40^{\circ} \mathrm{C}, 25^{\circ} \mathrm{C}, 85^{\circ} \mathrm{C}\right)$.

\subsection{Results of Neural Network Model}

As shown in Figure 29. to Figure 31, the neural network model accurately reflected the nonlinearity of the EPB system, where the differences between the actual output and 'Neural Network Force' were within $5 \mathrm{kgf}$ in all

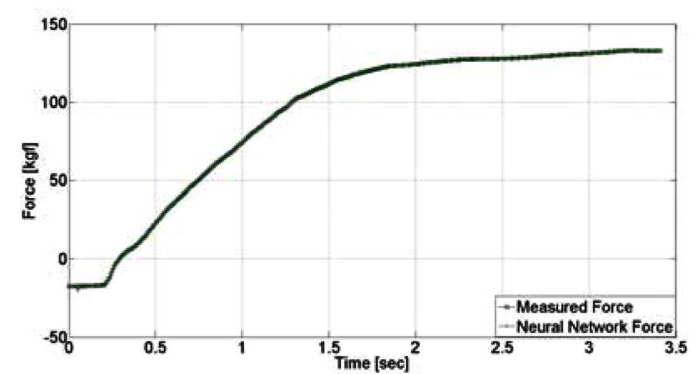

Figure 29. Results of measured force and neural network model $\left(-40^{\circ} \mathrm{C}\right)$.

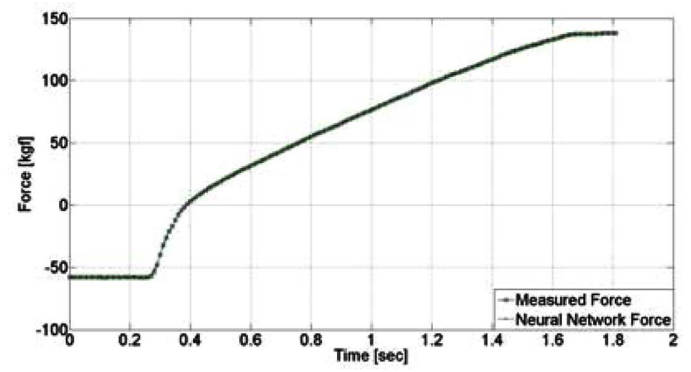

Figure 30. Results of measured force and neural network model $\left(25^{\circ} \mathrm{C}\right)$.

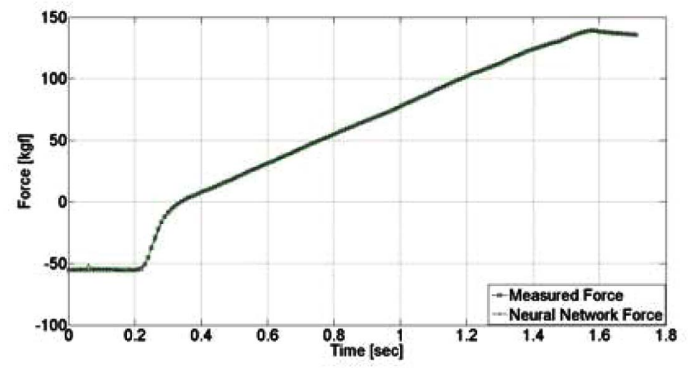

Figure 31. Results of measured force and neural network model $\left(85^{\circ} \mathrm{C}\right)$. operation sections and various temperature conditions. Learning should be conducted several times to obtain the results that reflect the nonlinearity of the EPB system. The weakness of neural network learning was that the same results could not be obtained every time because the computational time and the minimum number of learning trials could not be known.

3.5. Results of Parallel Model-based Fault Detection Algorithm

Figure 32, Figure 34, and Figure 36 shows the experimental results for the measured forces of the parking force sensor having sensor fault and the mathematical model, the fuzzy model, and the neural network model, respectively. The fault status of parking force sensor was as follows in this experiment. The output of parking force sensor reached to

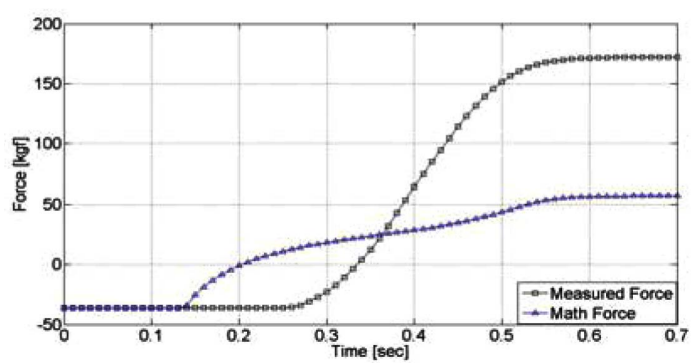

Figure 32. Results of measured force and mathematical model for force sensor fault.
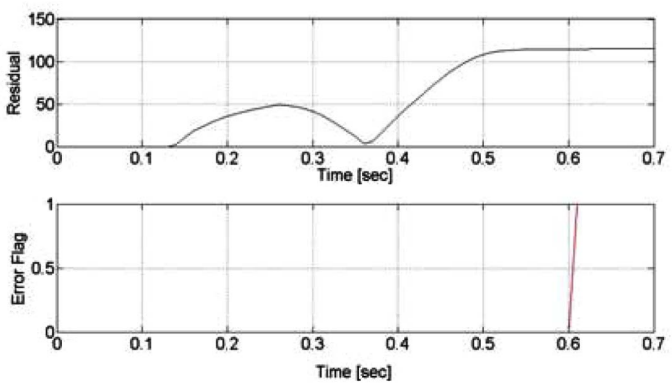

Figure 33. Results of residual and error flag of mathematical model for force sensor fault.

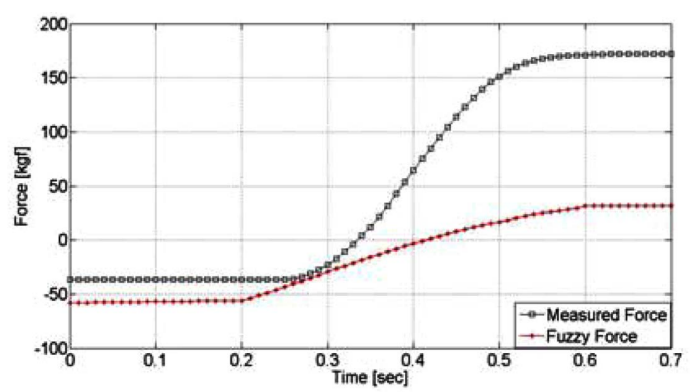

Figure 34. Results of measured force and fuzzy model for force sensor fault. 

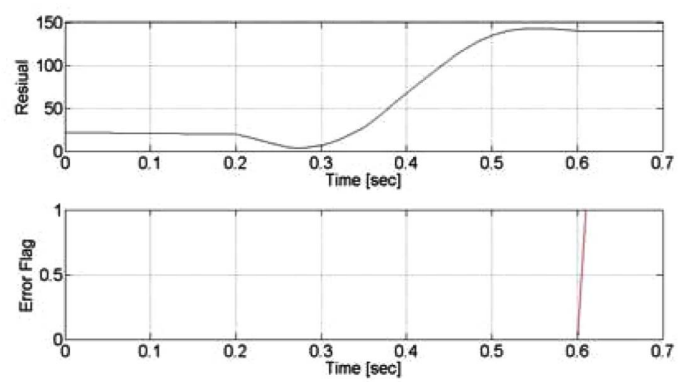

Figure 35. Results of residual and error flag of fuzzy model for force sensor fault.

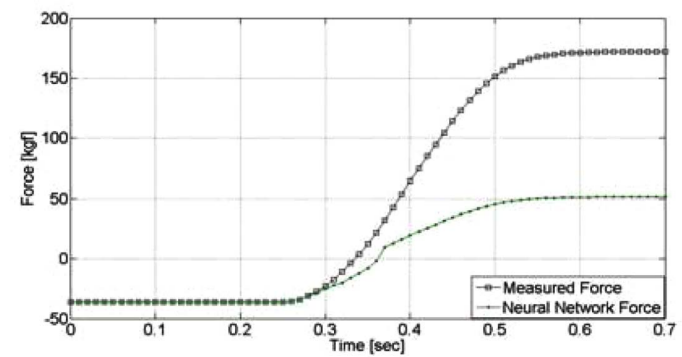

Figure 36. Result of measured force and neural network model for force sensor fault.
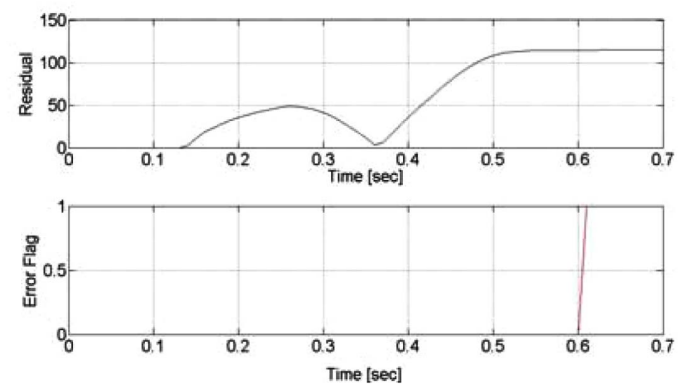

Figure 37. Results of residual and error flag of neural network model for force sensor fault.

the applied target force by $0.6 \mathrm{sec}$, which was $1.0 \mathrm{sec}$ faster than the normal state, and then the control was ended. In addition, the increasing slope for the output of parking force sensor was $100 \mathrm{kgf} / 100 \mathrm{~ms}$, which was approximately six times higher than the normal state $(15 \mathrm{kgf} / 100 \mathrm{~ms})$. As shown in Figure 33, Figure 35, and Figure 37, the error flag was occurred at $0.6 \mathrm{sec}$ because the parking control was terminated at this point and the value of residual exceeded the predefined threshold value stated in Table 4.

\subsection{Analysis of the Proposed Fault Detection Method} As shown in Figure 38, for the existing multi-modal based fault detection methods, fault detection condition applied to the EPB system at room temperature was to detect faults if the error that exceeds $35 \%$ of threshold occurred more than

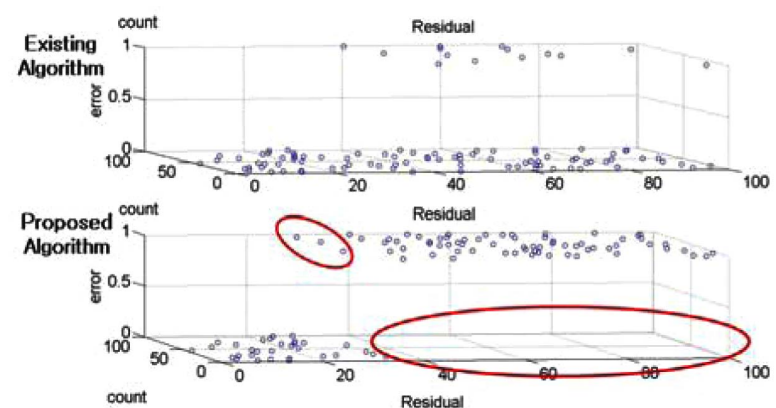

Figure 38. Performance comparison between existing and proposed fault detection algorithms.

three times(Fennel and Ding, 2000). In contrast, fault detection conditions of the proposed parallel-model based fault detection method were if the error that exceeds $35 \%$ of threshold occurred one time and the error that exceeds $25 \%$ to $35 \%$ of threshold occurred more than three times. Thus, the proposed parallel-model based fault detection method was able to detect faults when the error exceeded threshold for only one or two times as shown in Figure 38. Since the proposed method was able to detect faults when the error exceeded threshold for only one time, it could detect faults faster than the conventional methods and within the tolerance zone $(25 \%$ to $35 \%)$, which was not able to detect by the conventional methods.

\section{CONCLUSION}

The parallel model-based fault detection algorithm was proposed, which is an essential technology in commercializing the EPB system with parking force sensor. In order to increase the reliability of fault detection in the EPB system, the proposed fault detection algorithm used three models: mathematical model, fuzzy model, and neural network model. It could be applied to the system, which has only one sensor in the model. Furthermore, to minimize false error detection, the proposed fault detection algorithm was based on adaptive thresholds and the number of operations. It was able to integrate the algorithm to the developed EPB failsafe system to make the system more effective.

The proposed method was able to detect faults when the error exceeded threshold for only one time. Significantly, it could detect faults faster than the conventional methods and within the tolerance zone (25\% to $35 \%$ ), which was not able to detect by the conventional methods. Through these results, it can be inferred that the proposed model-based fault detection algorithm is a highly reliable fault detection algorithm.

As a future work, it is expected to improve the performance of fault detection for each of mathematical, fuzzy, and neural network model through further experiments. As the accuracy of fault detection increases, it 
is then expected to carry out research that detects the failure by using only one of the proposed fault detection models (Peng and Li, 2009).

ACKNOWLEDGEMENT-This research was supported by Technology Innovation Program of the Knowledge economy (No. 10041834, 10041351 ffunded by the Ministry of Knowledge Economy (MKE, Korea) and Basic Science Research Program through the National Research Foundation of Korea (NRF) funded by the Ministry of Education, Science and Technology (No. 2012R1A1A 2043822).

\section{REFERENCES}

Albas, E., Arikan, T. and Kuzkaya, J. C. (2001). In-process motor testing results using model-based fault detection approach. Proc. Elect. Insulation Conf. and Elect. Manufacturing and Coil Winding Conf., 643-647.

Altinten, A., Erdgan, S., Hapglu, H. and Alpbaz, M. (2003). Control of a polymerization reactor by fuzzy control method with genetic algorithm. Comput. Chem. Eng., 27, 1031-1040.

Beard, R. V. (1971). Failure accommodation in linear systems through self-organization. Technical Report MVT-7 I-1, Man Vehicle Lab, MIT, Cambridge.

Bose, N. and Raghavan, I. (2005). Prediction of emissions using combustion parameters in a diesel engine fitted with ceramic foam diesel particulate filter through artificial neural network techniques. Int. J. Automotive Technology 6, 2, 95-105.

Boskovic, J. and Mehra, R. K. (1998). A multiple modelbased reconfigurable flight control system design. Proc. 37th IEEE Conf. Decision and Control, Tampa, FL, 4503-4508.

Choi, C. S., Tae, S. J., Kim, H. M., Cho, K. N., Moon, J. M., Kim, J. Y. and Kwon, H. J. (2005). Fuzzy algorithm for FDD technique development of system multi-air conditioner. Trans. KSME B 29, 11, 1220-1228.

Chung, H., Chun, J., Kwon, S. and Kim, K. (2008). Development and performance evaluation of single puller type electric parking brake (EPB). KSAE 2008 Annual Conf., 2202-2206.

Demuth, H., Beale, M. and Hagan, M. (2009). Neural Network Toolbox ${ }^{\text {TM }} 6$ User's Guide. MathWorks.

Fennel, H. and Ding, E. L. (2000). A model-based failsafe system for the continental TEVES electronic-stabilityprogram (ESP). SAE Automotive Dynamics \& Stability Conf., SAE Paper No. 2000-01-1635.

Fussel, D., Balle, P. and Isermman, R. (1997). Closed loop fault diagnosis based on a nonlinear process model and automatic fuzzy rule generation. IFAC Symp. Safeprocess '97, 1, 359-364.

Gopinathan, M., Boskovic, J. D. and Mehra, R. K. (1998). A multiple model predictive scheme for fault-tolerant flight control design. Proc. 37th IEEE Conf. Decision and Control, Tampa, Florida.

Han, K., Huh, K., Hong, D., Kim, J., Kang, H. and Yoon, P. (2008). Real-time model-based fault diagnosis system for EHB system. Trans. KSAE 16, 4, 173-178.

Iida, O., Sato, M., Kiguchi, M., Iwamura, T. and Fukumura, S. (1988). Blast Furnace Control by Artificial Intelligence. Artificial Intelligence in Real-time Control. Rodd, M. G. and Suski, G. J. edn. Swansea. Pergamon Press. UK. 73.

Isermann, R. and Balle, P. (1997). Trends in the application of model-based fault detection and diagnosis of technical process. Control Eng. Practice 5, 5, 709-719.

Jung, H. G., Lee, Y. H., Kim, B. J., Yoon, P. J. and Kim, J. (2007). Stereo vision-based forward obstacle detection. Int. J. Automotive Technology 8, 4, 493-504.

Maybeck, P. S. and Stenvens, R. D. (1991). Reconfigurable flight control via multiple model adaptive control methods. IEEE Trans. Aerospace and Electron. Syst. 27, 3, 470-479.

Mihov, G., Dimitrov, E. and Nenov, N. (2004). Temperature errors compensation of force sensor for railway carriages wheel load measuring. 27th Int. Spring Seminar, 486-490.

Moon, B. and Park, C. (2009). Robust model based fault detection of EPB system for varying temperature. Trans. $K S A E$ 17, 5, 26-30.

Moon, B.-J., Kim, D.-H. and Park, C.-K. (2009). Fuzzy model-based fault detection method of EPB system for varying temperature. J. Control, Autom. and Syst. Eng. 15, 10, 1009-1013.

Patton, R. J. and Chen, J. (1994). Fault diagnosis in nonlinear dynamic systems via neural networks. Proc. IEE Int. Conf. Control, 2, 1346-1351.

Patton, R. J. and Willcox, S. W. (1987). Parameter insensitive technique for aircraft sensor. fault analysis. $J$. Guidance, Control, and Dynamics 10, 3, 359-367.

Peng, Y.-Q. and Li, W. (2009). Research on fuzzy control strategies for automotive EPB system with AMESim/ simulink co-simulation. 2009. Chinese Control and Decision Conf. (CCDC 2009), 1707-1712.

Pfeufer (1997). Application of model based fault detection and diagnosis to the quality assurance of an automotive actuator. Control Eng. Practice 5, 5, 703-708.

Sorsa, T. (1991). Neural networks in process fault diagnosis. IEEE Trans. Syst. Man and Cybern. 21, 4, 815-825.

Sorsa, T. and Koivo, H. (1993). Dynamic fault diagnosis using radial bas is function networks. TOOLDIAG '93, Toulouse, 160-169.

Sreedhar, R., Fernandez, B. and Masada, G. Y. (1995). A neural network based adaptive fault detection scheme. Proc. IEEE Amer. Control Conf., Seattle, Washington, 3259-3263. 